\title{
Diagnóstico De La Producción De Textos Descriptivos En Inglés
}

\author{
Rojas Castro Carmita Eulalia \\ Logroño Becerra Mónica Alejandra \\ Lara Olivo Lenin Iván \\ Yumi Guacho Lorena Maribel
}

Magister en Lingüística y Didáctica de la Enseñanza de Idiomas Extranjeros

Docente de la Escuela Superior Politécnica de Chimborazo, Centro de

Idiomas, Ecuador

Doi:10.19044/esj.2018.v14n32p29ＵRL:http://dx.doi.org/10.19044/esj.2018.v14n32p29

\begin{abstract}
The objective of the current research was to diagnosis the descriptive texts production as complement of the communicational skill in English, from the students of third level "M" from the Language Center from Escuela Superior Politécnica de Chimborazo in October 2016 - March 2017. For this reason, an intervention plan was applied with an evaluation rubric, to an intentional sample or a control group composed of 1 teacher and 41 students. The rubric was verified and validated by academic peers, who ratified the fulfilment of writing stages such as, prewriting, drafting, review, editing, and proofreading. The intervention lasted eight weeks, where the students carried out a set of activities that were qualified and quantified through a checklist with a weighted range of options. The research is descriptive, field and bibliographic. The information obtained was tabulated, analyzed and interpreted; the same determined the current scenario of the students in each of the stages for the descriptive texts production, obtaining as a conclusion about that a strategy is needed to influence on the production of descriptive texts.
\end{abstract}

Keywords: Diagnosis, Production of descriptive texts. English.

\section{Resumen}

El objetivo de la investigación fue diagnosticar la producción de textos descriptivos como complemento de la destreza comunicacional en inglés, de los estudiantes de tercer nivel paralelo " $M$ " de inglés del Centro de Idiomas de la Escuela Superior Politécnica de Chimborazo en el período Octubre 2016 Marzo 2017. Para lo cual, se aplicó un plan de intervención con una rúbrica de evaluación a una muestra intencionada que representa el grupo de control 
compuesta por 1 docente y 41 estudiantes. La rúbrica fue verificada y validada por pares académicos, quienes ratificaron el cumplimiento de las etapas de la escritura tales como: pre-escritura, borrador, revisión, edición y corrección. La intervención se aplicó en ocho semanas, donde los estudiantes realizaron un conjunto de actividades que fueron calificadas, mediante una lista de cotejo con un rango ponderado de opciones. La investigación es descriptiva, de campo y bibliográfica. La información obtenida fue tabulada, analizada e interpretada, y determinó el escenario de los estudiantes en cada una de las etapas para la producción de textos descriptivos, llegando a la conclusión que se necesita una estrategia para incidir en la producción de textos descriptivos.

Palabras clave: Diagnóstico, Producción de textos descriptivos. Inglés

\section{INTRODUCCIÓN}

Los y las estudiantes de las diferentes carreras de la Escuela Superior Politécnica de Chimborazo (ESPOCH), cursan seis niveles de inglés en la mayoría de carreras de pregrado, en estos niveles desarrollan estrategias de lectura, habla, escucha y escritura progresivamente en los que se incluyen la comprensión y creación de textos en inglés los dos últimos niveles donde se desarrollan la escritura como parte complementaria de su destreza comunicativa.

Gracias al conocimiento de las reglas básicas del idioma inglés; el resultado de la producción escrita suele aplicarse a partir del tercer nivel, al momento de transmitir ideas, escribir un párrafo o texto. Lo que hace que su producción, creación, expresión y pensamiento en otro idioma sean aspectos aún limitados. Debido a que no tienen una guía acerca de cómo iniciarse en el proceso de escritura. Es decir, no realizan una lluvia de ideas en la que se planifica previamente el texto, no llevan a cabo un primer borrador del texto, por lo tanto no lo revisan, corrigen y peor aún lo comparten con el resto de sus compañeros.

Por otro lado los docentes, a pesar de conocer la importancia de cada una de las destrezas en el proceso enseñanza- aprendizaje de una segunda lengua, suele incluir a breves rasgos la escritura dentro de la planificación de algunos temas básicos de enseñanza que se encuentran en el sílabo institucional, tanto por la complejidad que implica evaluar un trabajo escrito, como por la cantidad de estudiantes que asiste a cada nivel. Debido a la gran demanda de estudiantes que la ESPOCH recibe cada semestre en todas las facultades y carreras.

En la Escuela Superior Politécnica de Chimborazo, no existe una regulación en cuanto al uso o no de un texto básico, lo que representa hasta cierto punto una ventaja, ya que esto evidencia claramente la necesidad de implementar nuevas estrategias metodológicas, que ayuden a mejorar el 
desarrollo de la escritura ya que hacerlo apropiadamente, ha sido, es y será uno de los más grandes retos dentro de la formación profesional de los y las estudiantes.

Según (Lenneberg, 1967) notó que la especie humana aprende naturalmente a caminar y a hablar, pero que escribir y nadar son comportamientos aprendidos culturalmente de forma específica. Aprendemos a nadar si nuestro cuerpo está en el agua y alguien nos enseña, de la misma manera aprendemos a escribir si somos parte de una sociedad alfabetizada y alguien nos enseña, por eso hay personas que no saben nadar, otras que nadan con dificultad y otras que son excelentes nadadores y nadadoras, de forma similar sucede en la escritura. Para consolidar esta hipótesis, se hicieron algunas investigaciones alrededor de diversas teorías que básicamente confluyen en: Se llega al proceso de escritura por medio del sentido común, lo que permite planificar el escrito, hacer el borrador, revisarlo y corregirlo para tener como resultado escritos de diversa índole. Elbow (1973).

Algunos estudiantes que escriben, llegan con cierta predisposición sobre cómo organizar sus escritos en su lengua nativa, lo que dificulta que su escritura se adapte a las normas del idioma que estén aprendiendo. Kaplan (1966).

En estudios realizados a finales de los 70 se asume que la composición en lengua nativa y el aprendizaje de otra lengua eran similares, sino idénticas, la diferencia está en que suelen ser menos precisas por lo tanto se cometen más errores, por esta razón el profesor cuando enseña escritura debe manejar las diferencias lingüísticas y socioculturales.

La tarea de escribir se asume en base al estudio realizado por John Flower y Linda Hayes en la década de los 80, el presente estudio se basa en la inquietud de cómo funcionan los procesos mentales de algunos escritores mientras escriben, dando como resultados entre otros que en primera instancia se debe determinar qué se va escribir, para qué, a quién está dirigido el texto, qué clase de lenguaje debemos usar, el tiempo que tenemos para escribirlo, y cómo lo difundiremos. También se añade a la construcción del mismo, la información personal que tenemos acerca de algo, como cuando escuchamos una palabra y ésta nos trae a la mente diversas ideas, sensaciones y emociones, pues no basta sólo con plasmar nuestra experiencia sino que debemos acudir a la información bibliográfica, la misma que nos proveerá de datos certeros al respecto.

En última instancia tenemos el proceso de escritura en sí, determinado por tres etapas importantes que son: la planificación, traducción y revisión; dentro de la planificación se encuentra la generación de ideas y el ordenamiento de las mismas, La primera etapa nos dará una visión general acerca de lo que queremos escribir. La etapa de traducción, es lo que llamamos el borrador, ya que aquí detallamos lo planificado anteriormente. Y la última 
etapa incluye la revisión que aunque la mayoría de escritores no lo llevan a cabo es importante, porque aquí se hace una revisión de las ideas importantes que comúnmente omitimos, además de revisar si lo que estuvo planteado inicialmente se ha cumplido o no, si el orden de ideas es el apropiado y si lo que queremos exponer es correcto o erróneo.

En lo académico, profesional e investigativo, escribir es una representación textual (Sánchez, 2011) por medio de una construcción de sentido en la descripción de experiencias e interacciones sociales. También, es una práctica regulada por culturas escritas particulares, como los centros de educación superior, los grupos de investigación y las revistas académicas, entre otros escenarios. En el proceso de enseñanza - aprendizaje universitario, quienes escriben son los docentes y estudiantes, los investigadores y profesionales que trabajan con lenguajes de articulación social, dentro de ellas el idioma inglés.

La tarea de escribir es un acto profundo de reflexión, pensar antes de hacerlo requiere de un compromiso moral, lógico y social, un texto escrito ejerce la gran influencia de cambiar la mentalidad del lector en todos los aspectos. Esa es la función del escritor, hacer reflexionar, cambiar, dar vida a través del texto, en el caso de nuestro texto descriptivo el permitirle al lector un dibujo mental, lo más cercano a la realidad acerca del tema descrito.

La teoría del texto de Teun Van Dijk (1977) que fue inicialmente inspirada bajo la Teoría de la Gramática Generativa de Noam Chomsky, luego de muchas investigaciones propone posteriormente que a más de comprender las relaciones de significación entre las oraciones, que definen la coherencia, existen también relaciones entre todo lo que una oración indica, de allí nace la importancia de la noción de coherencia en cualquier teoría semántica del discurso y la idea obvia de que los textos también están organizados en niveles generales de descripción.

Teun Van Dijk menciona que los estudios hechos sobre el análisis del discurso deben ser diversos y en ellos se deben integrar los ámbitos relevantes de la lingüística, la pragmática, la sicología y las ciencias sociales permitiendo que contribuyan a un análisis crítico de la sociedad y haciendo que los y las estudiantes logren demostrar su pensamiento crítico, es decir que a más del verdadero uso de la lengua que incluye la variación social y dialectal, debe estudiarse también en términos de los actos del habla. Todos los estudios realizados en este ámbito son relevantes porque nos permiten acercarnos a un análisis completo de la teoría del texto, la misma que nos servirá para comprender de mejor manera el proceso que vamos a seguir con el fin de obtener un texto descriptivo como producto final de nuestra intervención.

(Dijk, 1977) propone como argumentos para su teoría, la existencia de las propiedades gramaticales más allá del límite de la oración, es decir 
establece relaciones semánticas entre oraciones, esto con el fin de comprender el texto en sí.

A través de la gramática textual se puede establecer relaciones entre otras teorías del estudio del discurso como la estilística, la narrativa, la poética, etc... para concluir, de acuerdo a la teoría de Van Dijk las oraciones y textos pueden funcionar a nivel discursivo como enunciados y discursos, es necesario establecer una definición de texto y discurso: Entendiendo como texto al conjunto de elementos lingüísticos, organizados según reglas de construcción y como discurso a la emisión concreta de un texto por parte de un enunciador, en un contexto determinado.

Por eso es imperativo, iniciar desde la pre-escritura, tomando la lluvia de ideas como estrategia única en este paso, luego continuar con los pasos de la escritura para estructurar un texto, pero antes veremos cómo se clasifican los textos con el fin de relacionar la producción del texto descriptivo y sus características.

La producción de textos descriptivos requiere de preguntas generadoras de ideas para su producción planteándose dos preguntas que pueden ayudar en el proceso de descripción textual:

¿Qué describe el párrafo? descripción?

¿Qué procedimientos tuvo que recorrer el autor para llegar a esta

Rojas (2011), indica además que "Para seleccionar rasgos es útil formular preguntas: ¿Cómo es? ¿Qué partes tiene? ¿De qué color es? ¿Para qué sirve? En fin, es necesario aplicar todos los sentidos sobre el objeto, lo que permitirá llevar al texto diversas imágenes: visuales, auditivas, olfativas, táctiles y gustativas". Y para este objetivo uno de los mayores recursos es el conocimiento y la aplicación de vocabulario, específicamente el uso de adjetivos.

De acuerdo a la teoría de Inteligencias Múltiples (Gardner, 1993) se involucran todos los sentidos (herramienta imprescindible en la producción de los textos descriptivos) dentro del proceso de aprendizaje, éste será más productivo; desarrollando también la inteligencia emocional, ya que se comprende de mejor manera sus sentimientos propios y los de los otros lo que a su vez permite comprender las etapas del comportamiento humano, desarrollando empatía y fortaleciendo la inteligencia interpersonal.

\section{METODOLOGÍA}

El enfoque de la investigación fue descriptivo, con el fin de analizar mediante una rúbrica de evaluación la situación de los estudiantes para la producción de textos descriptivos en inglés. Por ello, se buscó los argumentos que sustenten la aplicabilidad de estrategias o un método que permitan a la 
producción de textos descriptivos, de acuerdo a las necesidades que los y las estudiantes tienen en el desarrollo de la escritura en inglés.

Para ello, se requirió que la información sea analizada de forma cuantitativa, a través de la medición de parámetros en un rango numérico, cuyos resultados se sometieron a análisis e interpretación estadístico.

La población y muestra estudiada para la aplicación de la intervención, está constituida por 41 estudiantes de tercer nivel, hombres y mujeres de edades entre 18 y 21 años del Centro de Idiomas que forman parte del grupo de control, matriculados en el período Octubre 2016 - Marzo 2017 y que son estudiantes de diferentes facultades, escuelas y carreras de la Escuela Superior Politécnica de Chimborazo.

La investigación mediante un plan de intervención realizó una encuesta aplicada a la muestra, que justificó la importancia de la aplicación de un método para el desarrollo de la producción de textos descriptivos.

La investigación es exploratoria, ya que es un problema poco conocido, que intenta levantar la base para aplicar un método innovador, diferente y promisorio en el Centro de Idiomas de la Escuela Superior Politécnica de Chimborazo, con la expectativa de que se acoja como línea base para aplicar un método o estrategia de la producción de textos descriptivos, lo que contribuirá al cambio y permitirá corregir el problema investigado en el ambiente en el cual se desarrolló, con todas sus características, fortalezas y deficiencias.

El estudio además, corresponde a una investigación de campo, ya que para realizarla ha sido posible acceder a la información requerida desde el lugar en el que se manifiestan los hechos, (Centro de Idiomas de la Escuela Superior Politécnica de Chimborazo) siendo esta premisa vital, para la obtención de datos reales.

Además, es de carácter documental por el análisis de la información escrita del sujeto de estudio y seccional o transversal porque se la va a llevar a cabo con una serie de datos únicos obtenidos, en ocho semanas comprendidas entre el Lunes 17 de Octubre hasta el miércoles 7 de diciembre de 2016.

Para realizar la investigación se realizó un cronograma para que las cinco primeras semanas se desarrollen un conjunto de actividades con diversos temas requeridos, a fin de desarrollar el proceso de escritura de textos descriptivos y posteriormente realizar la respectiva evaluación mediante parámetros cuantitativos que permitan valorar y conocer el rendimiento académico de los estudiantes del tercer nivel. Así los y las estudiantes deberán emplear todas las posibles herramientas del idioma, para recrear el personaje, el objeto o el lugar de acuerdo a lo planificado.

Para la evaluación de la producción de textos descriptivos en inglés, se estableció una rúbrica de evaluación que considera los siguientes reactivos 
o etapas tales como: Pre- escritura (brainstorming), Borrador (Draft), Revisión (Revising), Edición (Editing), Corrección (proof-reading).

Las técnicas utilizadas en la investigación fueron básicamente de análisis de información, a través de encuesta realizada a través de los instrumentos de análisis documental, sistematización, análisis e interpretación de resultados a través de graficos de pastel y de barras. Mientras que los instrumentos son un test de escritura o prueba de diagnóstico, cuestionario con escala de medición a docentes.

Con la finalidad de determinar el conocimiento de los y las estudiantes sobre la producción de los textos descriptivos, se realizó una prueba de diagnóstico, dirigida a los y las estudiantes del tercer nivel del Centro de Idiomas, la misma se llevó a cabo en la Escuela Superior Politécnica de Chimborazo, que consta de las siguientes preguntas:

1. Draw a graphic organizer in which you are going to plan a descriptive text for the following picture.

2. Describe the picture in 8 lines (minimum) with different characteristics and use the correct punctuation.

3. Read your text again and underline the introduction, circle the body and put the conclusion into brackets.

4. Explain if the theme is related to the description.

5. Review and write your text again, but add some omitted important aspects.

Para verificar las exigencias técnicas de la validez y confiabilidad, se sometió el instrumento a 3 expertos pares académicos. Los instrumentos que constan en este proceso de intervención, son confiables ya que se orientan a los objetivos planteados y los resultados nos conducirán a plantear una propuesta capaz de aplicarse a la realidad del Centro de Idiomas de la Escuela Superior Politécnica de Chimborazo.

Para el cálculo de la validez, el test se sometió al criterio de expertos respecto a la producción de textos aplicando el IVC (índice de validez del contenido):

$$
I V C=\frac{n e-\frac{N}{2}}{\frac{N}{2}}
$$

ne $=$ Número de expertos que consideran válido el ítem analizado

$\mathrm{N}=$ Número total de expertos consultados 
Tabla 1. Cálculo de validez

\begin{tabular}{|c|c|c|c|c|c|}
\hline Reactivo & EXP. 1 & EXP. 2 & EXP. 3 & VALIDOS & $\begin{array}{c}\text { ÍNDICE DE } \\
\text { VALIDEZ }\end{array}$ \\
\hline Pre-writing & SI & SI & SI & 3 & 1 \\
\hline Drafting & SI & SI & SI & 3 & 1 \\
\hline Revising & SI & SI & SI & 3 & 1 \\
\hline Editing & SI & NO & SI & 2 & 0,33 \\
\hline Proofreading & SI & SI & NO & 2 & 0,33 \\
\hline
\end{tabular}

Elaborado por: Rojas Carmen, 2018

Se define como confiabilidad al grado en que su aplicación repetida al mismo sujeto u objeto produce resultados iguales (consistentes y coherentes). Para validar el instrumento, se empleará el índice de confiabilidad de Crombach:

Dónde:

$$
\alpha_{e s t .}=\frac{k p}{1+p(k-1)}
$$

$\mathrm{k}=\mathrm{al}$ número de ítems del instrumento

$\mathrm{p}=$ Promedio de correlación lineal de los ítems calculado con la siguiente fórmula:

$$
\begin{gathered}
p=[k(k-1)] / 2 \\
p=10 \\
\alpha_{\text {est. }}=\left(10^{*} 5\right) /(1+5(10-1)) \\
\alpha_{\text {est. }}=1,22 \\
\alpha_{\text {est. }}=1,22>1,00
\end{gathered}
$$

$1,22>1$ (Confiabilidad positiva)

El valor de alpha es 1,22 y es mayor que 1; por tanto la confiablidad de la encuesta es positiva.

\section{RESULTADOS}

Se presentan a continuación los resultados de las evaluaciones obtenidas de los 41 estudiantes del 3er nivel del Centro de Idiomas de la Escuela Superior Politécnica de Chimborazo. Los puntajes obtenidos son el resultado de la rúbrica para evaluar la producción de textos descriptivos en inglés.

Tabla 1. Ponderación de opciones de reactivos

\begin{tabular}{lc}
\hline RÚBRICA & EQUIVALENCIA \\
\hline EXCELLENT & 2,0 \\
VERY GOOD & 1,6 \\
GOOD & 1,2 \\
FAIR & 0,8 \\
POOR & 0.2 \\
\hline
\end{tabular}

Fuente: Rúbrica de evaluación Elaborado por: Rojas Carmita, 2017 
A través de un check se utilizó la rúbrica para evaluar la actividad propuesta, para luego ponderar mediante una hoja de cálculo y obtener la evaluación total obtenida por cada estudiante.

Cada estudiante fue evaluado mediante una tarea a fin de obtener su calificación sobre 10 puntos. Se tabuló la información obtenida con el objetivo de registrar el impacto por cada una de las opciones de los 41 estudiantes. Finalmente se presentó las evaluaciones que han sido obtenidas por cada estudiante.

Tabla 2: Frecuencias totales de los elementos de la rúbrica

\begin{tabular}{ccccccc}
\hline RÚBRICA & EXCELLENT & VERY GOOD & GOOD & FAIR & POOR & TOTAL \\
\hline Pre-writing & 1 & 3 & 10 & 11 & 16 & 41 \\
Drafting & 2 & 2 & 7 & 11 & 19 & 41 \\
Revising & 3 & 5 & 8 & 15 & 10 & 41 \\
Editing & 1 & 2 & 7 & 17 & 14 & 41 \\
Proofreading & 0 & 4 & 5 & 15 & 17 & 41 \\
\hline
\end{tabular}

Fuente: Rúbrica de evaluación

Elaborado por: Rojas Carmita, 2017

Reactivo 1: Brainstorming (Preescritura)

Tabla 3: Frecuencia del reactivo 1

\begin{tabular}{cccccc}
\hline RÚBRICA & EXCELLENT & VERY GOOD & GOOD & FAIR & POOR \\
\hline Pre-writing & 1 & 3 & 10 & 11 & 16 \\
\hline \multicolumn{5}{c}{$\begin{array}{r}\text { Fuente: Rúbrica de evaluación } \\
\text { Elaborado por: Rojas Carmen, } 2017\end{array}$} \\
\hline \multicolumn{4}{c}{}
\end{tabular}

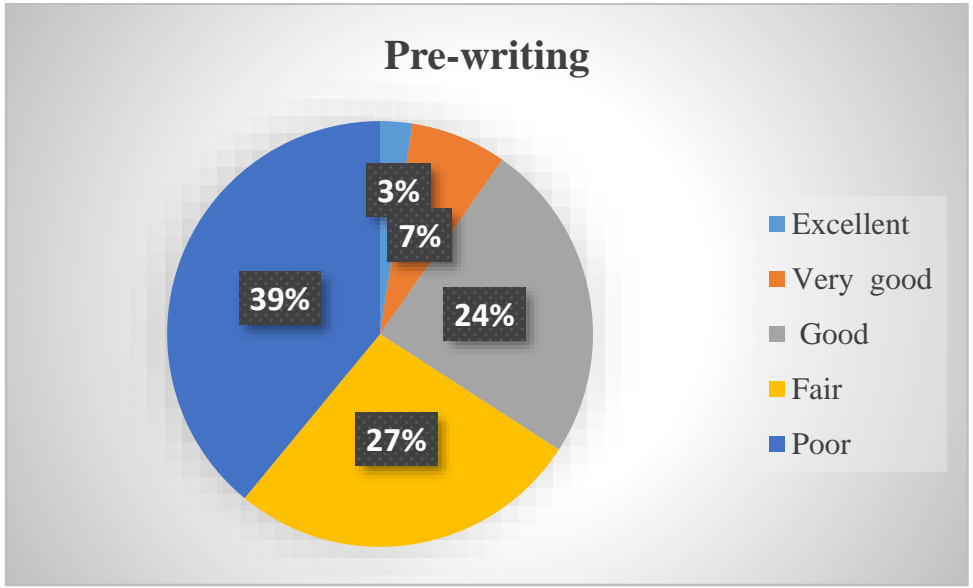

Gráfico 1: Frecuencia del reactivo 1

\section{Análisis e interpretación de datos}

Del 100\% de los estudiantes del tercer nivel del paralelo "M" evaluados en cuanto a la Pre-escritura del Grupo control, el 39\% registró que no realizaron correctamente; el 27\% razonable, el 24\% Bueno; el 7\% Muy bueno; 
mientras que el 3\% registró excelente. Es decir que en la mayoría de los estudiantes la etapa de Pre-escritura, en la producción de textos descriptivos en Inglés no fue aceptable.

Reactivo 2: Drafting (Redacción)

Tabla 4: Frecuencia del reactivo 2

\begin{tabular}{cccccc}
\hline RÚBRICA & EXCELLENT & VERY GOOD & GOOD & FAIR & POOR \\
\hline Drafting & 2 & 2 & 7 & 11 & 19 \\
\hline
\end{tabular}

Fuente: Rúbrica de evaluación

Elaborado por: Rojas Carmita, 2017

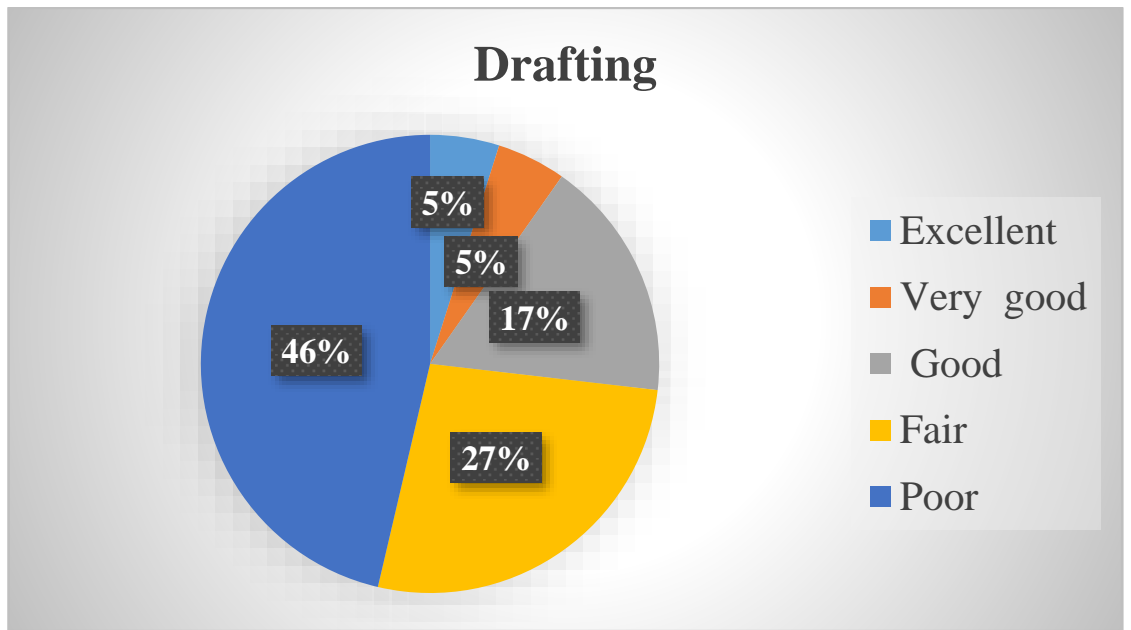

Gráfico 2: Frecuencia del reactivo 2

Análisis e interpretación de datos

Del $100 \%$ de los estudiantes del tercer nivel paralelo " $M$ " evaluados en cuanto al borrador inicial, el $46 \%$ registró un borrador desfavorable, el $27 \%$ aceptable, el 17\% Bueno, el 5\% Muy Bueno, mientras que el 5\% registro que su desempeño en esta actividad fue Excelente. Por tanto, la mayoría de los estudiantes mostraba un nivel desfavorable en cuanto al borrador inicial en el proceso de escritura para la producción de textos descriptivos en Inglés y necesita ser influenciada por un método diferente.

Reactivo 3: Revising (Revisión)

Tabla 5: Frecuencia del reactivo 3

\begin{tabular}{cccccc}
\hline RÚBRICA & EXCELLENT & VERY GOOD & GOOD & FAIR & POOR \\
\hline Revising & 3 & 5 & 8 & 15 & 10 \\
\hline
\end{tabular}

Fuente: Rúbrica de evaluación

Elaborado por: Rojas Carmita, 2017 


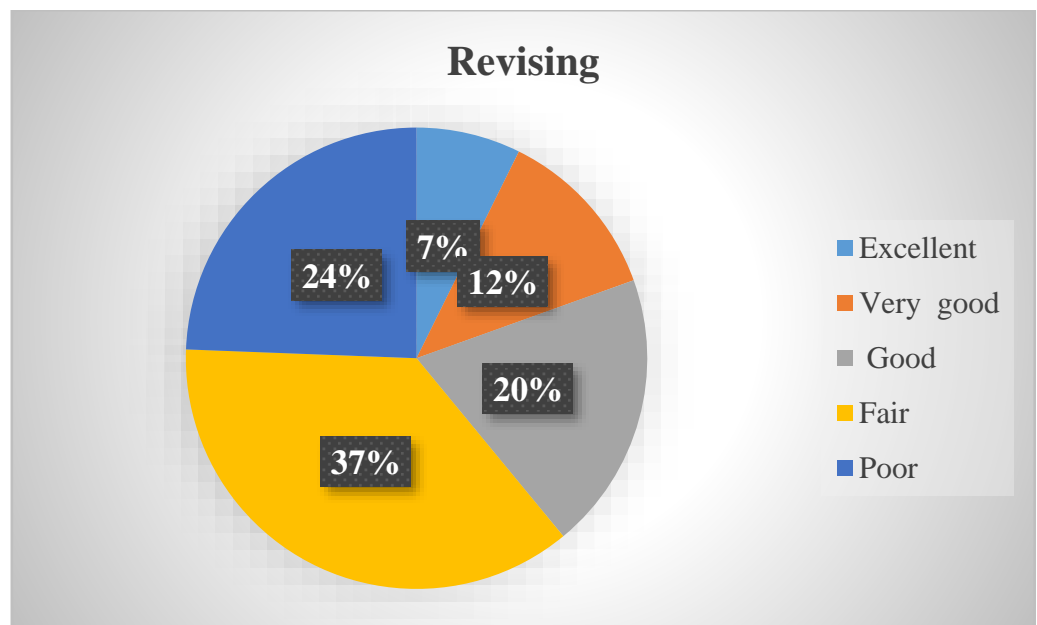

Gráfico 3: Frecuencia del reactivo 3

Análisis e interpretación de datos

Del 100\% de los estudiantes del tercer nivel del paralelo "M" evaluados en cuanto al elemento de la rúbrica Revisión, el $37 \%$ fue aceptable; el $24 \%$ tenía una revisión desfavorable, el $20 \%$ tenía buena revisión; el $12 \%$ muy buena; mientras que el restante $7 \%$ registro que era excelente. Es decir que no se estaba realizando una correcta revisión al momento de escribir textos descriptivos en Inglés, la misma que necesita mejorar.

Reactivo 4: Editing (Edición)

Tabla 6: Frecuencia del reactivo 4

\begin{tabular}{cccccc}
\hline RÚBRICA & EXCELLENT & VERY GOOD & GOOD & FAIR & POOR \\
\hline Editing & 1 & 2 & 7 & 17 & 14 \\
\hline \multicolumn{5}{c}{ Fuente: Rúbrica de evaluación } \\
Elaborado por: Rojas Carmita, 2017
\end{tabular}

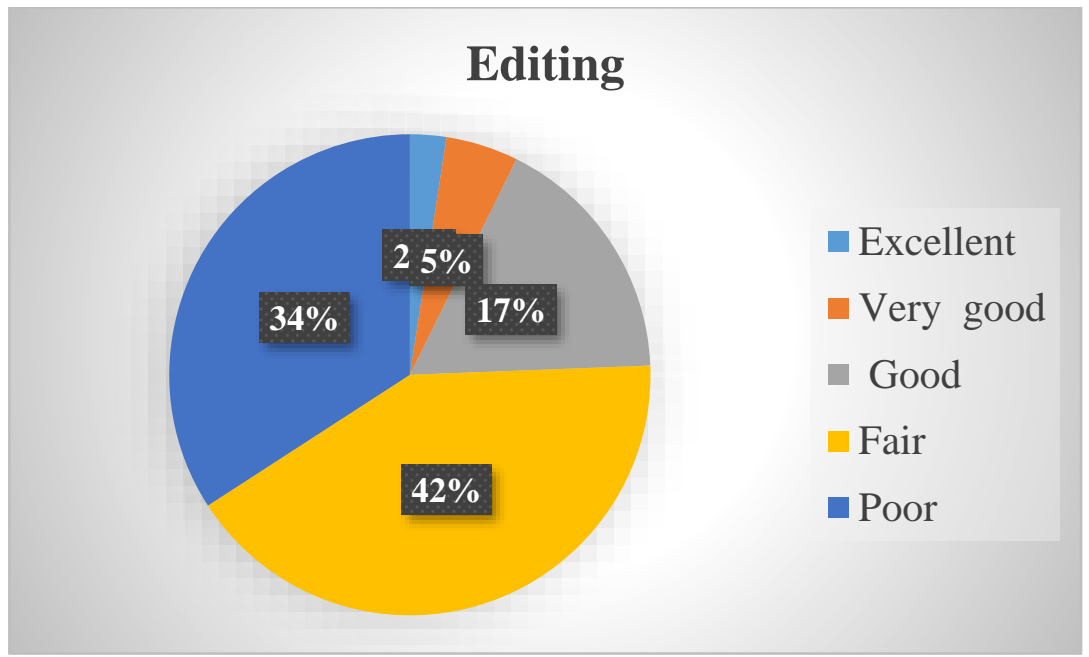

Gráfico 4: Frecuencia del reactivo 4

Análisis e interpretación de datos 
Del $100 \%$ de los estudiantes del tercer nivel paralelo "M" evaluados en cuanto a la Edición en el del Grupo control, el $42 \%$ es aceptable; el $34 \%$ es deficiente, el 17\% es Bueno; el 5\% Muy bueno; mientras que el restante 2\% registró Excelente. Por lo que se necesita que el proceso de escritura en Inglés sea influenciado, pues las frecuencias negativas son más notorias.

Reactivo 5: Proofreading

Tabla 7: Frecuencia del reactivo 5

\begin{tabular}{cccccc}
\hline RÚBRICA & EXCELLENT & VERY GOOD & GOOD & FAIR & POOR \\
\hline Proofreading & 0 & 4 & 5 & 15 & 17 \\
\hline
\end{tabular}

Fuente: Rúbrica de evaluación

Elaborado por: Rojas Carmita, 2017

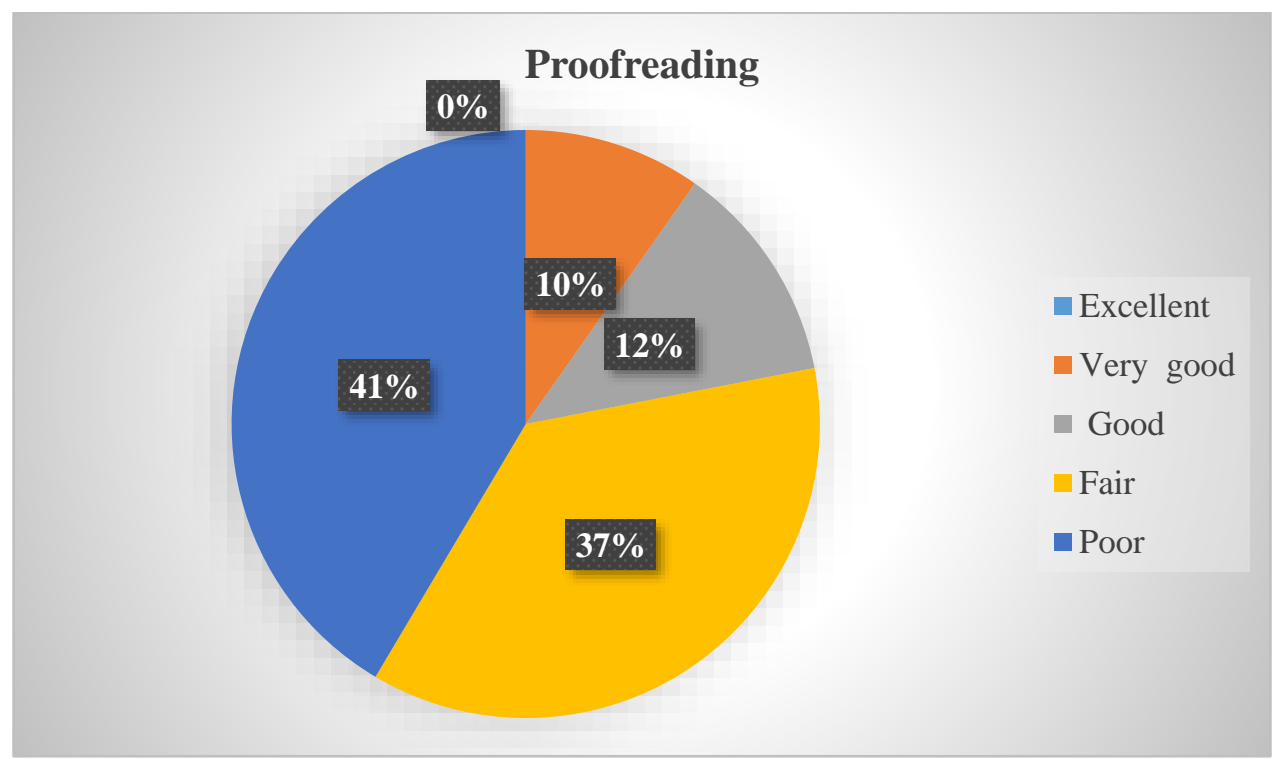

Gráfico 5: Frecuencia del reactivo 5

Análisis e interpretación de datos

Del 100\% de los estudiantes del tercer nivel del paralelo "M" evaluados en cuanto a la Corrección de los textos descriptivos en el del Grupo control, el $41 \%$ es deficiente; el $37 \%$ es aceptable, el 12\% Buena; el 10\% registró Muy buena; mientras que no se registra frecuencias de la opción excelente. Por lo que se necesitó que la producción de textos descriptivos en Inglés sea influenciada. 


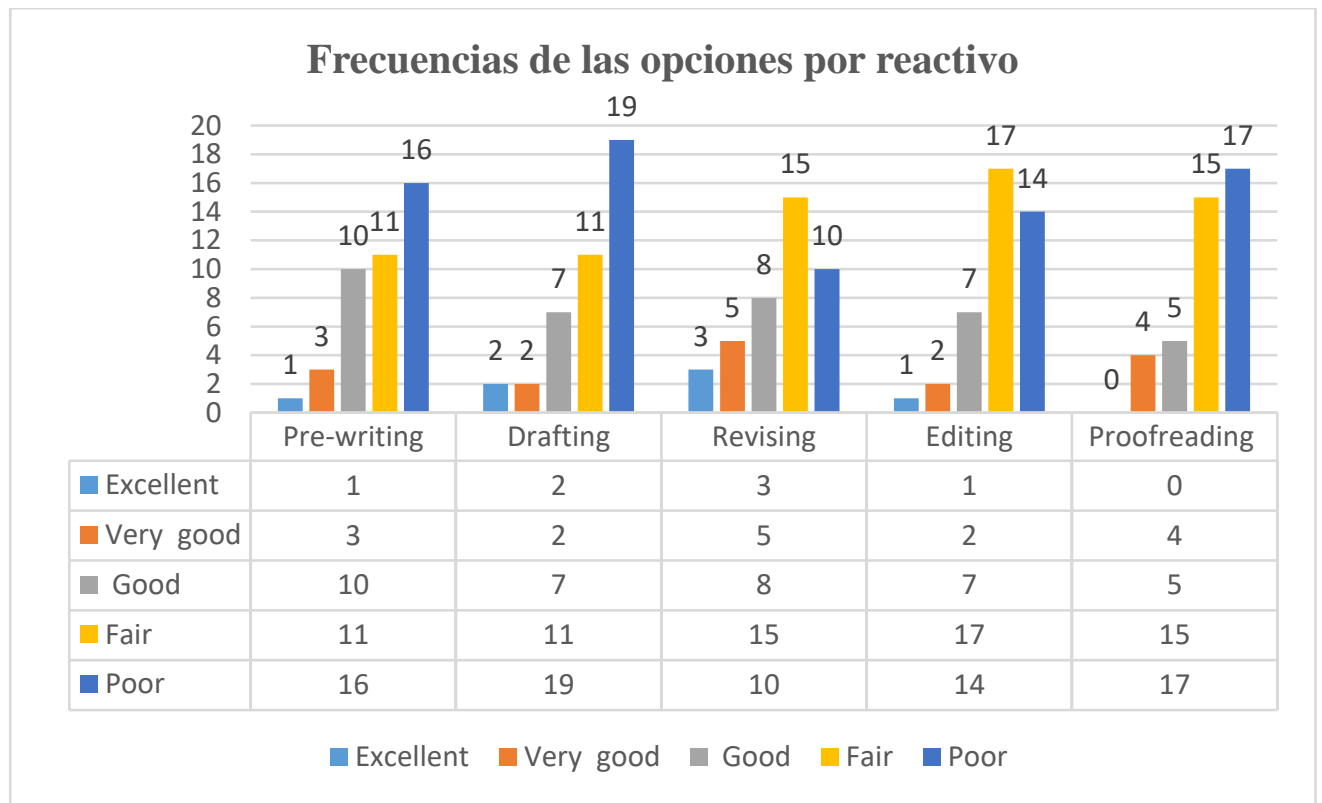

Gráfico 6: Frecuencias de las opciones de los elementos de la rúbrica

\section{Análisis e interpretación}

Se puede observar que las opciones de deficiente y aceptable son las que más frecuencia registraron en los elementos de la rúbrica y representan el estado inicial de evaluación en el grupo control.

\section{CONCLUSION}

El análisis de los reactivos, evidencia que existen problemas en las diferentes en el proceso de escritura de textos descriptivos; es decir, que se presentan deficiencias en la aplicación de cada una de las etapas, que son sistemáticas y complementarias para llegar al objetivo. Propiciando un escenario para que se pueda trabajar y desarrollar trabajos colaborativos para asumir roles complementarios para la construcción colectiva de los textos. De la misma manera, se requiere que el docente sea el guía explique y evalúe paso a paso las etapas de escritura pues los estudiantes se pueden retroalimentar y pueda corregir errores en la marcha.

En la producción de los textos descriptivos se determinó que no se aplicaba la escritura como un proceso, sino más bien como una actividad sin lineamientos, pasos o etapas específicas, las que a su vez necesitan el tiempo suficiente para ser explicadas y aplicadas.

El análisis de los resultados obtenidos durante este proyecto de intervención, requiere el planteamiento de una propuesta con estrategias, métodos o actividades que estén dirigida tanto a docentes como estudiantes, 
para mejorar la producción de textos descriptivo en el Centro de Idiomas de la ESPOCH.

\section{References:}

1. Djick, Teun Van (1977). "La pragmática de la comunicación literaria", Ms. En español. Universidad de Amsterdam.

2. Elbow, P. (1973). Writing without Teachers, pp. 12-75. New York, NY: Oxford University Press.

3. Gardner, H. (1993). Multiple Intelligences. The Theory in Practice. A Reader. Nueva York, EUA: Basic Books.

4. John Hayes - Psychology. (s.f.). En Carnege Mellon University. Recuperado el 23 de Abril del 2016. Disponible en: http://www.cmu.edu/dietrich/psychology/people/coretrainingfaculty/hayes-john.html

5. Linda Flower. (s.f.). En Wikipedia. Recuperado el 23 de Abril del 2016. Disponible en: https://en.wikipedia.org/wiki/Linda_Flower

6. Kaplan Robert B, Vai Ramanathan (1996). Audience and voice in current L1 composition texts: Some implications for ESL student writers, Journal of Second Language Writing, 5, 1, (21).

7. Lenneberg, E. H. (1967): Biological Foundations of Language, John Wiley and Sons Inc.

8. Rojas, V. (2011). Competencias en la comunicación: hacia las prácticas del discurso. (E. Ediciones, Ed.). Bogotá. 